\title{
Competencias TIC en los docentes de las unidades tecnológicas de Santander
}

\author{
ICT competences in the teachers of the Technological Units of Santander
}

\author{
Ernesto Solano Hernández \\ Unidades Tecnológicas de Santander \\ esolano@correo.uts.edu.co \\ Victoria I. Marín Juarros \\ Carl von Ossietzky Universität Oldenburg (Alemania) \\ victoria.marin@uni-oldenburg.de \\ Alba Rossi Rocha Vásquez \\ Unidades Tecnológicas de Santander \\ arocha@correo.uts.edu.co
}

Recibido: $1 / 10 / 2018$

Aceptado: $11 / 12 / 2018$

Publicado: 21/12/2018

\section{RESUMEN}

Las nuevas tecnologías de la información y la comunicación se convirtieron en el eje fundamental de la sociedad del siglo XXI, generando nuevos espacios formativos y modalidades de formación. La práctica docente debe hacerse partícipe de los nuevos modelos de aprendizaje y formación, para lo cual surge la necesidad de la cualificación docente en términos de competencia digital, un argumento que se solicita con fuerza incluir en esta actividad. En este artículo se describen y presentan los resultados de la investigación realizada en las Unidades Tecnológicas de Santander (UTS, Colombia), para conocer, evaluar y pronosticar el nivel de conocimiento y competencia digital del personal docente, adscrito a las dos facultades de la institución; dentro de las dimensiones evaluadas se solicitó información referente a datos de identificación (sociodemográficos y laborales), uso y conocimiento de TIC, integración de las TIC en la práctica docente, formación docente e innovación en TIC y el componente ético en la labor docente. Se dispuso de un instrumento de investigación (encuesta) ya utilizado y validado en otro estudio del mismo tipo, que se adaptó al contexto institucional de las UTS. Se utilizó un servidor de la universidad para alojar la encuesta y que fuera presentada de forma telemática por los profesores. Como conclusión del análisis de resultados, se diseñó una propuesta de trabajo para la mejora de las competencias digitales basados en las necesidades de formación detectadas.

\section{PALABRAS CLAVE}

Competencias Docentes; competencia digital; evaluación; profesorado universitario; formación del profesorado.

\section{ABSTRACT}

The new information and communication technologies became the fundamental axis of 21 st century society, generating new training spaces and training modalities. The teaching practice must become a participant in the new models of learning and training, for which the need arises for the teaching 
qualification in terms of digital competence, an argument that is strongly requested to include in this activity. This article describes and presents the results of the research carried out in the Santander Technology Units (UTS, Colombia), to know, assess and forecast the level of knowledge and digital competence of teaching staff, assigned to the two faculties of the institution ; Within the evaluated dimensions, information was requested regarding identification data (sociodemographic and labor), use and knowledge of ICT, integration of ICT in teaching practice, teacher training and innovation in ICT and the ethical component in teaching work. There was a research instrument (survey) already used and validated in another study of the same type, which was adapted to the institutional context of the UTS. A university server was used to host the survey and was presented telematically by the teachers. As a conclusion to the analysis of results, a work proposal was designed to improve digital skills based on the training needs detected.

\section{KEYWORDS}

Teaching Competencies; digital competence; evaluation; university teaching staff; teacher training

\section{CITA RECOMENDADA}

Solano, E., Marín, V. y Rocha A.R. (2018). Competencias TIC en los docentes de las unidades tecnológicas de Santander. RIITE. Revista Interuniversitaria de Investigación en Tecnología Educativa, 5, 67-83. Doi: http://dx.doi.org/10.6018/riite/2018/344231

\section{Principales aportaciones del artículo y futuras líneas de investigación:}

- El artículo presenta un tema actual en el ámbito de la Educación y las Tecnologías de la Información y Comunicación (TIC).

- Autopercepción del nivel de apropiación de competencia digital docente en una institución de educación superior colombiana

- Como futura línea de investigación, la influencia de la propuesta de mejora en los docentes, luego de su implementación y desarrollo, para poder verificar el nivel de apropiación de estas competencias

\section{INTRODUCCIÓN}

Los nuevos entornos formativos, incorporan las tecnologías de la información y la comunicación (TIC) y conceptos subyacentes, que aportan en cierta manera valor agregado a la nueva forma de interacción de las personas con la información para aprovechar los procesos de enseñanza mediados por TIC (Solano, Rocha, \& Marín, 2016).

Las TIC, utilizadas en estos nuevos espacios formativos, llegan como apoyo al docente con posibilidades de utilización e incorporación de nuevas herramientas, ampliando la oferta e integración educativa, eliminando las barreras espacio temporales y generando una nueva relación profesor-estudiante. La competencia digital, se refiere a la capacidad, el conocimiento y la actitud que se posee sobre el uso de las TIC en los diferentes escenarios de aplicación. El Parlamento Europeo define la competencia digital de la siguiente manera: "La competencia digital entraña el uso seguro y crítico de las tecnologías de la sociedad de la información (TSI) para el trabajo, el ocio y la comunicación. Se sustenta en las competencias básicas en materia de TIC: el uso de ordenadores para obtener, evaluar, almacenar, producir, presentar e intercambiar información, y comunicarse y participar en redes de colaboración a través de Internet" (Parlamento Europeo, 2006, p.15).

De acuerdo con Cerverá, Martínez y Mon (2015), el concepto de competencia digital nace como una nueva visión del aprendizaje y los estudios formales donde se consolidan destrezas y conocimientos de una persona o individuo como objetivo en el desarrollo de sus estudios, esto es nuevas habilidades técnicas y cognitivas en un ambiente tecnológico, comunicativo e informacional. 
Estamos inmersos en una sociedad digital y además trabajamos en ella, desde la universidad existe la responsabilidad en los docentes de adquirir las competencias específicas, para afrontar la nueva sociedad del conocimiento; el docente universitario no debe ser ajeno a este nuevo rol y lo exhorta a adquirir este nuevo conjunto de competencias TIC, replicarlas en el desarrollo del proceso formativo, para que logre diseñar experiencias de aprendizaje significativas donde los estudiantes sean el punto central del proceso, a través de la utilización de las TIC.

Según Pozuelo (2014), el cambio de paradigma o mentalidad es un escenario donde surgen retos y oportunidades, en la actualidad internet es una de la principales fuentes de información y de capacitación no solo para profesores sino para cualquier individuo que pretenda hacer parte de estos nuevos entornos formativos, por lo que se espera que las instituciones hagan parte de este nuevo contexto; no se concibe un docente que pertenezca a esta era digital y que no utilice las TIC en su práctica docente, por lo cual es importante actualizarse no solo en su ámbito disciplinar, sino también en el aspecto informático para que ejerza de una forma responsable y competente.

El contenido de este artículo presenta los resultados de un estudio realizado sobre competencia digital docente en las Unidades Tecnológicas de Santander, institución de educación superior del sector público ubicada en la ciudad de Bucaramanga-Colombia; cuenta con aproximadamente 17.000 estudiantes y 930 docentes repartidos en dos facultades y 4 regionales. El objeto de estudio fue conocer o establecer el estado real de las competencias digitales de los docentes adscritos a la institución; se utilizó un cuestionario ya validado en otro estudio como instrumento para la recolección de datos; de igual manera se presenta la parte metodológica utilizada, el paradigma en el cual se enmarcó el estudio; y como conclusión general, la propuesta de mejora frente a los resultados obtenidos en el estudio.

\section{ESTADO DEL ARTE}

De acuerdo con Rocha (2018), los docentes de hoy en su mayoría son inmigrantes digitales, mientras que los alumnos son nativos digitales, esto es, que mientras que los docentes sostienen un manejo de lo digital, partiendo de lo analógico, todavía compran libros en las tiendas especializadas, toman notas en papeles escritas a lápiz o lapicero y llevan agenda física o cuaderno. Los estudiantes viven inmersos en los desarrollos tecnológicos, todo lo bajan de la red, son usuarios complusivos de blogs, video tutoriales, wikipedia y las tareas son realizadas en medios digitales y llevadas en memorias USB o en la nube; esto hace necesario que los docentes cambien de mentalidad y adquieran un cierto nivel de competencia digital en el uso y manejo de las TIC; los docentes no deben ser ajenos a este nuevo rol, son partícipes de la nueva era digital y están llamados a cumplimentarlas no solo para la sobrevivencia en un proceso educativo cambiante sino para mejorarlo (Cerverá, 2013).

En la nueva era del conocimiento, es necesario realizar un análisis desde la perspectiva profesional, sobre las características que debe poseer un buen docente para que cumpla con sus tareas asignadas, realice un buen desempeño y obtenga resultados eficientes desde su práctica; esto, de acuerdo con Zabalza \& Beraza (2003) quienes afirman: "Lo que todos los profesores necesitamos es un conocimiento profesional de la docencia" (p.70) lo cual hace necesario que en el trabajo cotidiano de la docencia, se busque poseer o mejorar el conjunto de conocimientos y habilidades que se involucran en el desarrollo de la práctica docente.

De acuerdo con Rodriguez Espinar (2007) en Prendes (2010) el buen docente:

- reúne las suficientes actitudes y aptitudes para ejercer la actividad docente;

- requiere centrarse en el "saber hacer" del proceso formativo;

- debe mantener un buen comportamiento en el aula y en sus relaciones interpersonales;

- desarrolla en forma competente las tareas dentro y fuera del aula;

- se identifica por los resultados en el logro de los objetivos académicos planteados; 
- se comporta como un verdadero profesional en la dimensión ética y en la forma como presta sus servicios a la comunidad.

En el mundo se han llevado a cabo múltiples estudios para verificar el estado de la competencia digital de los docentes, en todos los niveles de estudio, ya sean primarios, secundarios o universitarios, refiriéndose a la capacidad que tienen los docentes para involucrarse en la adquisición y manejo de los conceptos referentes a las nuevas tecnologías de la información y comunicación TIC y de las herramientas y desarrollos que se generan a partir de ellas.

En el año 1997 la Organización para la Cooperación y el Desarrollo Económico (OCDE), planteó el proyecto DeSeCo (Definición y Selección de Competencias), cuyo objetivo era proporcionar el marco de conceptos básicos clave para fortalecer las evaluaciones de tipo internacional como las del informe PISA y la definición de objetivos para la formación permanente en los sistemas educativos, incluyendo el concepto de competencia afirmando que son las habilidades que unos individuos dominan mejor que otros (Hernández, Gamboa, \& Ayala, 2014).

El proyecto TUNING plantea como competencia general la sistémica; lo que destaca en cierta manera el uso de las nuevas tecnologías de la información siendo una de las competencias que los profesores deben poseer para el desempeño de su actividad docente (Prendes Espinoza \& Gutiérrez Porlan, 2013). El proyecto en el año 2000, construye una clasificación de competencias docentes genéricas y específicas para cada área temática. Durán (2014) enumera las de tipo genérico, que se describen a continuación:

- Competencias instrumentales: son competencias que incluyen habilidades cognoscitivas, destrezas tecnológicas y destrezas lingüísticas.

- Competencias interpersonales: son las capacidades individuales por las que las personas muestran facilidad en procesos de interacción social y la cooperación.

- Competencias sistémicas: son aquellas que se entrelazan entre la comprensión, la sensibilidad y el conocimiento para que el individuo vea las partes de un todo, cómo se relacionan y se agrupan; son básicas para la adquisición previa de las instrumentales e interpersonales.

Para realizar el análisis inicial del estado del arte sobre competencia digital docente e instrumentos de evaluación de la competencia digital se utilizaron tres herramientas para centrar o enfocar los resultados de los estudios: Google Académico, Redalyc, y Dialnet.

Las búsquedas realizadas con estas herramientas en su conjunto arrojaron alrededor de 35 trabajos que incluían las palabras clave "instrumentos de evaluación" y "competencia digital". Sólo se realizaron búsquedas en idioma español. Luego se analizó los siguientes elementos: título del trabajo, creadores, fecha de creación, institución de origen, grupo de investigación, tipo de documento, destinatarios del instrumento, validación del instrumento, ítems o dimensiones que se evalúan y, por último, la fuente o repositorio donde se encuentran los trabajos analizados.

La revisión pormenorizada de los elementos permitió evaluar cada unos de los ítem, en los trabajos y se seleccionaron 10 estudios que se enfocaban en competencia digital docente y que contara cada uno con un instrumento de evaluación, creado y validado o que hubiese sido adaptado de acuerdo al contexto, la Tabla 1, resume los 10 trabajos seleccionado:

Tabla 1. Estudios analizados como estado del arte

\begin{tabular}{|c|l|}
\hline \# ITEM & \multicolumn{1}{|c|}{ TíTULO } \\
\hline 1 & $\begin{array}{l}\text { Diseño y Validación de un instrumento de evaluación para la certificación de la } \\
\text { competencia TIC del profesorado universitario (Durán, 2014) }\end{array}$ \\
\hline 2 & $\begin{array}{l}\text { Competencias TIC para la docencia en la universidad pública Española: } \\
\text { Indicadores y propuestas para la definición de buenas prácticas (Prendes, 2010) }\end{array}$ \\
\hline
\end{tabular}




\begin{tabular}{|c|c|}
\hline 3 & $\begin{array}{l}\text { Competencia digital del profesorado de educación secundaria: Un instrumento de } \\
\text { evaluación (Torres, 2014) }\end{array}$ \\
\hline 4 & $\begin{array}{l}\text { Alfabetización digital en docentes de educación superior: construcción y prueba } \\
\text { empírica de un instrumento de evaluación (Rangel \& Peñaloza, 2013) }\end{array}$ \\
\hline 5 & $\begin{array}{l}\text { Instrumento de evaluación de competencias digitales para adolescentes en } \\
\text { riesgo social (Carrera \& Coiduras, 2012) }\end{array}$ \\
\hline 6 & $\begin{array}{l}\text { Diseño y validación de un instrumento para evaluar la competencia digital de los } \\
\text { docentes en la educación superior española (Agreda, Hinojo \& Sola, 2016) }\end{array}$ \\
\hline 7 & $\begin{array}{l}\text { Concepciones sobre las Tecnologías de la Información y la Comunicación (TIC y } \\
\text { sus implicaciones educativas: Un estudio exploratorio con profesorado de la } \\
\text { provincia chilena de Ñuble. (Puentes, Roig, Sanhueza, \& Friz,2013) }\end{array}$ \\
\hline 8 & $\begin{array}{l}\text { La formación del profesorado en tic y la socialización en el aula (Santos, Vega \& } \\
\text { Sanabria, 2014) }\end{array}$ \\
\hline 9 & $\begin{array}{l}\text { Competencias digitales en docentes de educación secundaria. Municipio de un } \\
\text { Estado del Noroeste de México. (Mortis, Valdés, Angulo, García, \& Cuevas, 2013) }\end{array}$ \\
\hline 10 & $\begin{array}{l}\text { Competencia digital docente: ¿dónde estamos? perfil del docente de educación } \\
\text { primaria. (Valdivieso \& González, 2016) }\end{array}$ \\
\hline
\end{tabular}

Durán (2014), Torres (2014), toman como base a Prendes (2010) y lo referencian en sus estudios y en la creación de los respectivos instrumentos de investigación, por esto, se tomaron en cuenta para la adaptación del instrumento en el contexto institucional de las Unidades Tecnológicas de Santander, porque fueron realizados a medida, es decir creados para un público específico como docentes universitarios o profesores de secundaria. Torres (2014) se tomó como base para la adaptación del instrumento, ya, que las dimensiones o categorías que evaluaba eran iguales a las que se iban a evaluar en este estudio.

\section{MÉTODO}

El estudio en mención, hace referencia al cuerpo docente de las Unidades Tecnológicas de Santander, aplicando un cuestionario de forma telemática con preguntas e indicadores que hacen referencia a competencia digital y a las nuevas tecnologías de la información y la comunicación; el objetivo general: "Elaborar una propuesta de trabajo para el mejoramiento de las competencias TIC en los docentes de las Unidades Tecnológicas de Santander (UTS), basados en las necesidades de formación".

Para lograr el propósito general de esta investigación se pretende cumplir con los siguientes objetivos específicos:

- Describir el estado de las competencias TIC en una muestra representativa de docentes.

- Conocer la percepción de los docentes sobre su nivel de conocimiento y uso en TIC.

- Identificar y establecer las necesidades de formación del cuerpo docente en competencia digital.

La población fue el profesorado adscrito a la institución, corresponde a 894 docentes como población total, de los cuales 141 son la muestra aceptante, representando el $15.77 \%$ del total de la población considerándola como representativa, el muestreo fue de tipo probabilístico, ya que el total de los docentes obtuvo la misma oportunidad de participar en la encuesta, para esto se contactó a cada uno de los docentes mediante el envío de un correo electrónico a cada una de sus respectivas cuentas electrónicas; para garantizar el anonimato y confidencialidad de los datos no se solicitó el número de identificación y se omitió la solicitud de nombre y apellidos. Los aceptantes fueron quienes contestaron el cuestionario en línea. 
El trabajo de investigación se realizó teniendo en cuenta que la posición del investigador es observador-externo, porque se utilizan mediciones controladas; el paradigma cuantitativo (desde fuera para buscar causas y explicaciones) de corte positivista por ser comparable, medible y replicable de acuerdo a Martínez (2013). Se utiliza el método descriptivo de tipo no experimental porque la relación de los eventos se han producido con anterioridad, así como para lograr concretar los objetivos específicos que son medir, conocer el uso y dominio, las actitudes y las percepciones sobre las TIC y su nivel de competencia digital; es necesario tener en cuenta que en los estudios descriptivos se mide o recolecta la información para describir lo que se investiga. Este método es importante cuando se realizan investigaciones sobre fenómenos educativos, porque no se limitan solo a la recogida de datos, sino que responden a situaciones sobre el estado de competencias digitales.

La recogida de datos se realizó utilizando un instrumento de investigación tipo encuesta, que no fue creado, sino adaptado de Torres (2014), Prendes (2010) y Durán (2014); según Blasco, Mengual, \& Roig (2007), que si el investigador localiza o establece un cuestionario o instrumento de investigación con validez y fiabilidad establecida, puede ahorrar tiempo y dinero para agilizar en cierta medida la investigación, de acuerdo a lo anterior se adaptó el instrumento de Torres (2014), que había sido validado y utilizado en el correspondiente estudio.

La adaptación consistió en mantener la estructura del cuestionario y realizar las modificaciones leves al contexto educativo de la institución, manteniendo en más del $90 \%$ las preguntas con su estructura. Se definieron 5 categorías o dimensiones para evaluar así:

- Datos de identificación

- Uso y conocimiento de TIC

- Integración de recursos tecnológicos en la práctica docente

- Formación docente e innovación en TIC

- Componente ético en la labor docente

El cuestionario se compone de 82 ítems que forman las 5 dimensiones o categorías, conformadas por preguntas de selección y opción múltiple (una 0 varias opciones de respuesta), dicotómicas, que contienen dos opciones de respuesta (sí-no, cierto-falso, masculino-femenino, etc.), aunque en algunos casos se adiciona una neutra (no sabe, no responde, N/A), como complemento para este tipo de preguntas; y escala Likert, que ofrecen una amplia gama de respuestas para que los profesores elijan. La escala Likert permite asignar un valor o puntaje a cada opción de respuesta, como medida de actitud, dentro de una escala continua con valoraciones entre 1 y 5 , normalmente, o con otro rango de valores o puntuación. en función de su posición frente a la afirmación sugerida por el ítem (Guil Bozal, 2005).

El instrumento se alojó en un servidor institucional del aplicativo Lime Survey, que es un gestor de encuestas básico y que permite el manejo de análisis estadístico de la información almacenada; sin embargo para el análisis de datos, se utilizó el paquete estadístico SPSS en su versión 22 para Windows. Con éste se realiza el primer análisis, midiendo el índice de consistencia interna, mediante el Alpha de Cronbach, el cual arrojó un resultado de 0,948, lo cual indica que el instrumento de medida puede considerarse "fiable"; porque un instrumento es confiable si el índice de consistencia interna es superior a 0,80 (Quero Virla, 2010). Luego del análisis de fiabilidad realizado al instrumento y los constructos que hacían parte de él, se procedió a realizar un análisis de tipo descriptivo, de cada una de la categorías establecidas en el cuestionario, y se realizaron las tablas de frecuencias de cada constructo.

\section{RESULTADOS}

La primera dimensión hace referencia a Datos de Identificación, también conocidos como datos etnográficos o sociodemográficos, que presentan el contexto institucional de la población, para este caso particular las Unidades Tecnológicas de Santander y su cuerpo docente.

Los resultados muestran que cerca del $50 \%$ de los encuestados pertenecen a un rango de edad entre 30 y 45 años con un grado de escolaridad de especialización y maestría, lo que 
indica un nivel de estudio considerablemente alto (ver Figura 1), si se tiene en cuenta el contexto Latinoamericano y del Caribe donde el nivel de estudios y formación posgradual está rezagado respecto al resto del mundo; aunque las cifras van en aumento, dista mucho del nivel de formación en otras regiones (Bellei, Poblete, Sepúlveda, Orellana, \& Abarca, 2013).

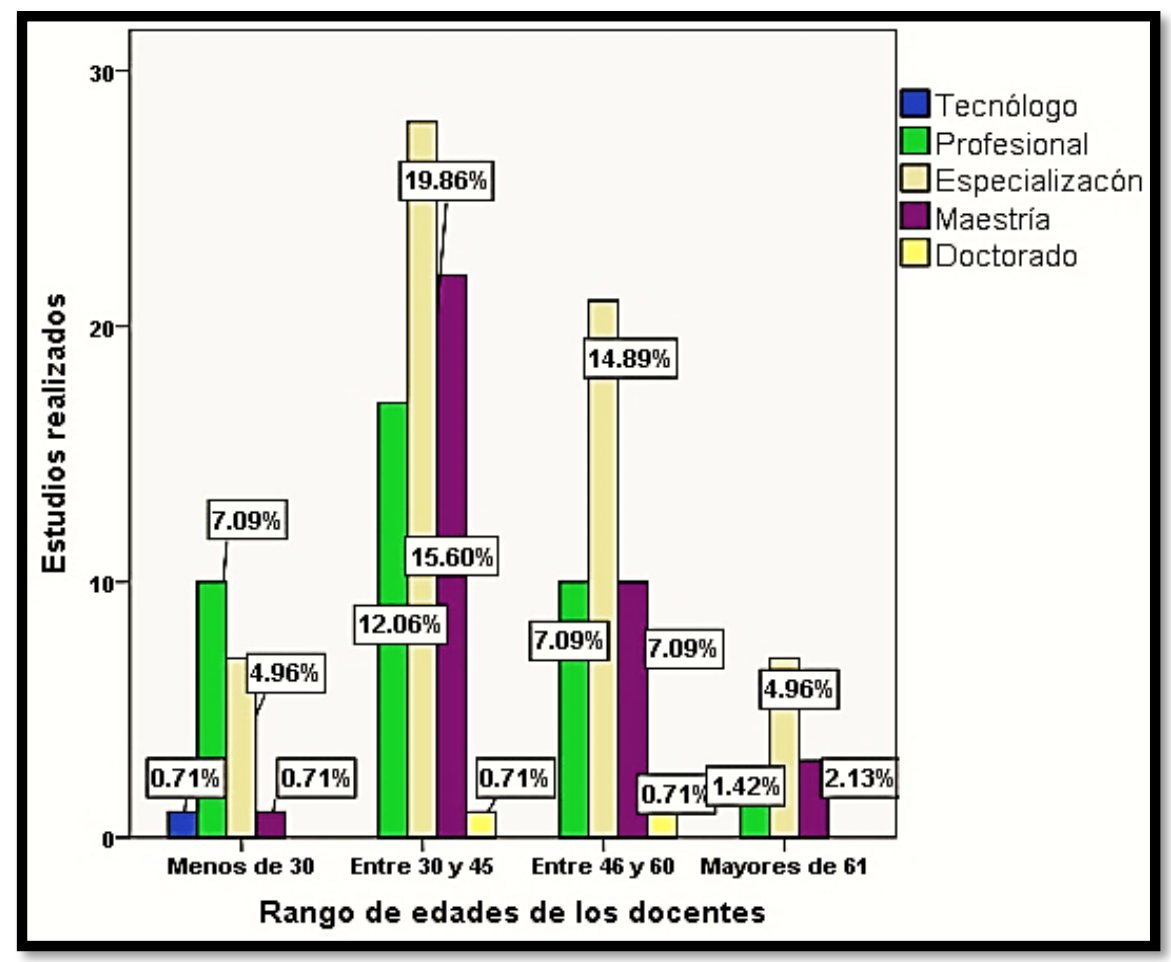

Figura 1. Estudios realizados por edades.

De igual forma se aprecia un alto porcentaje de profesores con contratación tipo cátedra, contrastando comparativamente con los docentes de tiempo completo o de carrera, representando un $70.92 \%$ de los docentes vinculados como se observa en la Figura 2. Estos resultados son consistentes con la contratación en la institución, a finales del año 2017 de 894 profesores, 226 son vinculados como docentes de carrera o docentes ocasionales de tiempo completo con nivel de maestria o doctorado, representando aproximadamente un $25 \%$ de los vinculados totales (Lengerke, 2017); mientras que el restante, son profesores cátedra de acuerdo al esquema de contratación actual representando la mayoría de docentes vinculados y se evidencia en los resultados observados en la Figura 2.



Figura 2. Tipo de vinculación. 
En la categoría de uso y conocimiento de TIC, se refiere un alto grado de conocimiento en actividades básicas de mantenimiento del PC,utilización de sistemas de protección de datos y antivirus en un porcentaje igual al $56.03 \%$, además resuelven en un alto porcentaje las incidencias técnicas que se presentan, así como el aprendizaje de forma autónoma, con resultados porcentuales cerca al $43 \%$.

El uso y conocimiento de foros, mensajería instantanea y redes sociales muestra porcentajes muy altos, debido en gran parte al buen manejo y conocimiento de plataformas como Facebook, WhatsApp, los servicios gratuitos de correo como gmail, yahoo y hotmail, sin embargo; el microbloging y los sistemas de videoconferencia son pocos conocidos y utilizados, lo que traduce desinteres o desconocimiento por las mismas, soportado en el hecho que el porcentaje de profesores supera los 40 años está por encima del $65 \%$ y deben adaptarse a la tecnología y a sus cambios; el trabajo colaborativo, el intercambio de archivos y los mundos virtuales mantienen la misma tendencia y se puede explicar por la misma razón, no son residentes digitales como afirma David White, sino visitantes digitales (Gisbert \& Esteve, 2011).

En cuanto a las aplicaciones de manejo de información, las herramientas de búsqueda, ocupan un lugar privilegiado, donde, google resalta como el buscador preferido, los docentes afirman poseer un conocimiento mayor sobre los editores de texto, editor de presentaciones, editores multimedia y el software específico utilizado por el profesor en su práctica, sin embargo; es importante anotar que estas herramientas referidas hacen parte de la suite ofimática de Microsoft y no a herramientas de la web 2.0 o 3.0 y se ve contrastado cuando se verifican los resultados generados sobre los marcadores sociales, las páginas de inicio personalizadas, las herramientas de curación de contenidos, que son poco o nada conocidos y mucho menos utilizados, la Tabla 2 muestra los valores de las frecuencias.

Tabla 2. Tabla de frecuencias

\begin{tabular}{|l|c|c|c|c|c|c|c|c|}
\cline { 2 - 9 } \multicolumn{1}{c|}{ INFORMACIÓN } & Nada & Poco & Bastante & Mucho & Nada & Poco & Bastante & Mucho \\
\hline $\begin{array}{l}\text { Herramientas de búsqueda (google, } \\
\text { bibliotecas de recursos,...) }\end{array}$ & $2.1 \%$ & $12.1 \%$ & $33.3 \%$ & $52.5 \%$ & $2.1 \%$ & $7.1 \%$ & $35.5 \%$ & $55.3 \%$ \\
\hline $\begin{array}{l}\text { Herramientas de publicación en red } \\
\text { (Flickr, blog, wiki, Slideshare..) }\end{array}$ & $8.5 \%$ & $38.3 \%$ & $27.7 \%$ & $25.5 \%$ & $16.3 \%$ & $39.7 \%$ & $24.1 \%$ & $19.9 \%$ \\
\hline $\begin{array}{l}\text { Marcadores sociales (Delicius, Mr. } \\
\text { Wong...) }\end{array}$ & $48.2 \%$ & $37.6 \%$ & $9.2 \%$ & $5 \%$ & $58.2 \%$ & $30.5 \%$ & $7.1 \%$ & $4.3 \%$ \\
\hline $\begin{array}{l}\text { Lectores de RSS. (RRSS Owl, } \\
\text { SAge...) }\end{array}$ & $51.1 \%$ & $35.5 \%$ & $9.2 \%$ & $4.3 \%$ & $59.6 \%$ & $30.5 \%$ & $5.7 \%$ & $4.3 \%$ \\
\hline $\begin{array}{l}\text { Páginas de inicio personalizadas } \\
\text { (netvibes, Symbaloo..) }\end{array}$ & $51.1 \%$ & $31.2 \%$ & $13.5 \%$ & $4.3 \%$ & $56.7 \%$ & $29.1 \%$ & $9.2 \%$ & $5 \%$ \\
\hline Lifestreaming (friendfeed) & $56 \%$ & $31.9 \%$ & $9.2 \%$ & $2.8 \%$ & $61.7 \%$ & $28.4 \%$ & $5.7 \%$ & $4.3 \%$ \\
\hline $\begin{array}{l}\text { Editores de texto (Microsoft Office } \\
\text { Word, Open Office Writer...) }\end{array}$ & $2.1 \%$ & $7.8 \%$ & $34 \%$ & $56 \%$ & $2.1 \%$ & $7.8 \%$ & $31.9 \%$ & $58.2 \%$ \\
\hline $\begin{array}{l}\text { Editor de presentaciones visuales } \\
\text { (Prezzi, Microsoft Office Power } \\
\text { Point...) }\end{array}$ & $0.7 \%$ & $14.9 \%$ & $34 \%$ & $50.4 \%$ & $1.4 \%$ & $15.6 \%$ & $31.9 \%$ & $51.1 \%$ \\
\hline $\begin{array}{l}\text { Editor multimedia (gráfico, imágenes, } \\
\text { audio, vídeo) }\end{array}$ & $4.3 \%$ & $26.2 \%$ & $38.3 \%$ & $31.2 \%$ & $4.3 \%$ & $33.33 \%$ & $33.33 \%$ & $29.1 \%$ \\
\hline $\begin{array}{l}\text { Editor de páginas web (Weebly, } \\
\text { Adobe Dreamweaver) }\end{array}$ & $29.8 \%$ & $39 \%$ & $19.9 \%$ & $11.3 \%$ & $39 \%$ & $36.9 \%$ & $14.9 \%$ & $9.2 \%$ \\
\hline $\begin{array}{l}\text { Software específico del ámbito de } \\
\text { trabajo }\end{array}$ & $4.3 \%$ & $17.7 \%$ & $44 \%$ & $34 \%$ & $9.9 \%$ & $18.4 \%$ & $36.9 \%$ & $34.8 \%$ \\
\hline $\begin{array}{l}\text { Herramientas para la curación de } \\
\text { contenidos (Scoop.it, Pinterest, etc.) }\end{array}$ & $45.4 \%$ & $35.5 \%$ & $9.9 \%$ & $9.2 \%$ & $50.4 \%$ & $34.8 \%$ & $6.4 \%$ & $8.5 \%$ \\
\hline
\end{tabular}

Fuente: elaboración propia

Respecto de la integración de recursos tecnológicos en la práctica docente, se evidencia que la mayor limitación de las TIC es el acceso a la red; aunque se realizan múltiples esfuerzos por mejorar la cobertura, es insuficiente y costosa, con bajos anchos de banda, en relación con otras instituciones. La institución cuenta con varias salas de informática al servicio de la comunidad educativa, estas son insuficientes porque son dedicadas en un gran 
porcentaje de su tiempo a las asignaturas que se imparten y la disponibilidad para la utilización por los docentes y alumnos es muy reducida, lo que no permite un libre desarrollo de actividades que involucren TIC, la Figura 3 presenta de forma gráfica los resultados sobre las limitaciones.

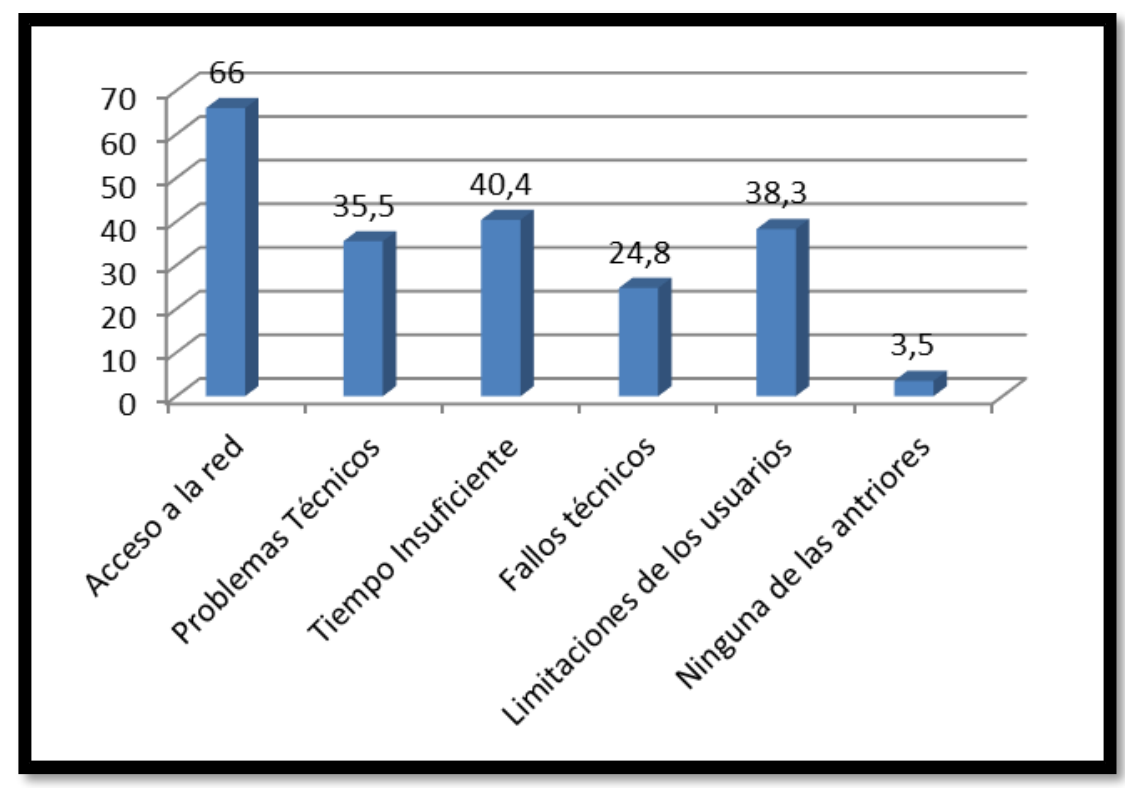

Figura 3. Limitaciones de las TIC

En cuanto a las posibilidades que poseen las TIC, el acceso a la información, la diversidad de metodologías y la flexibilización de tiempos y espacios son consideradas como las de mayor trascendencia en el proceso de enseñanza aprendizaje, generado esto por la facilidad que provee Internet para acceder a la información sin las limitaciones espacio temporales de la enseñanza tradicional.

Por su parte las estrategias metodológicas como el webquest y la caza del tesoro con porcentajes que bordean el $10 \%$ en ambas estrategias, refiriéndose a su conocimiento y uso, permitiendo afirmar el desconocimiento de las mismas; por el contrario el aprendizaje basado en estudio de casos, aprendizaje basado en problemas y el aprendizaje basado en proyectos obtienen porcentajes de uso y conocimiento por encima del $90 \%$ y la razón principal es que son las metodologías establecidas en la institución desde el macrocurrículo; la publicación de materiales didacticos apoyados en las herramientas TIC es muy baja así mismo la utilización de software libre y se evidencia poco o bajo acompañamiento de forma virtual con los estudiantes.

En la dimensión sobre formación docente e innovación en TIC es muy baja la realización de proyectos de innovación con el apoyo de las nuevas tecnologías, lo que indica bajo compromiso del docente de realizar actividades formativas, que estén apoyadas con estas herramientas tecnológicas debido al desconocimiento, lo que desencadena que prefieran aprender algo nuevo a través de cursos o jornadas de formación que realiza la institución de forma presencial, sin embargo en menor medida siguen videotutoriales en internet para aprender algo nuevo a través de la web, de hecho se evidencia el desconocimiento de herramientas tecnológica indispensables para el apoyo del proceso formativo, ver Figura 4. 


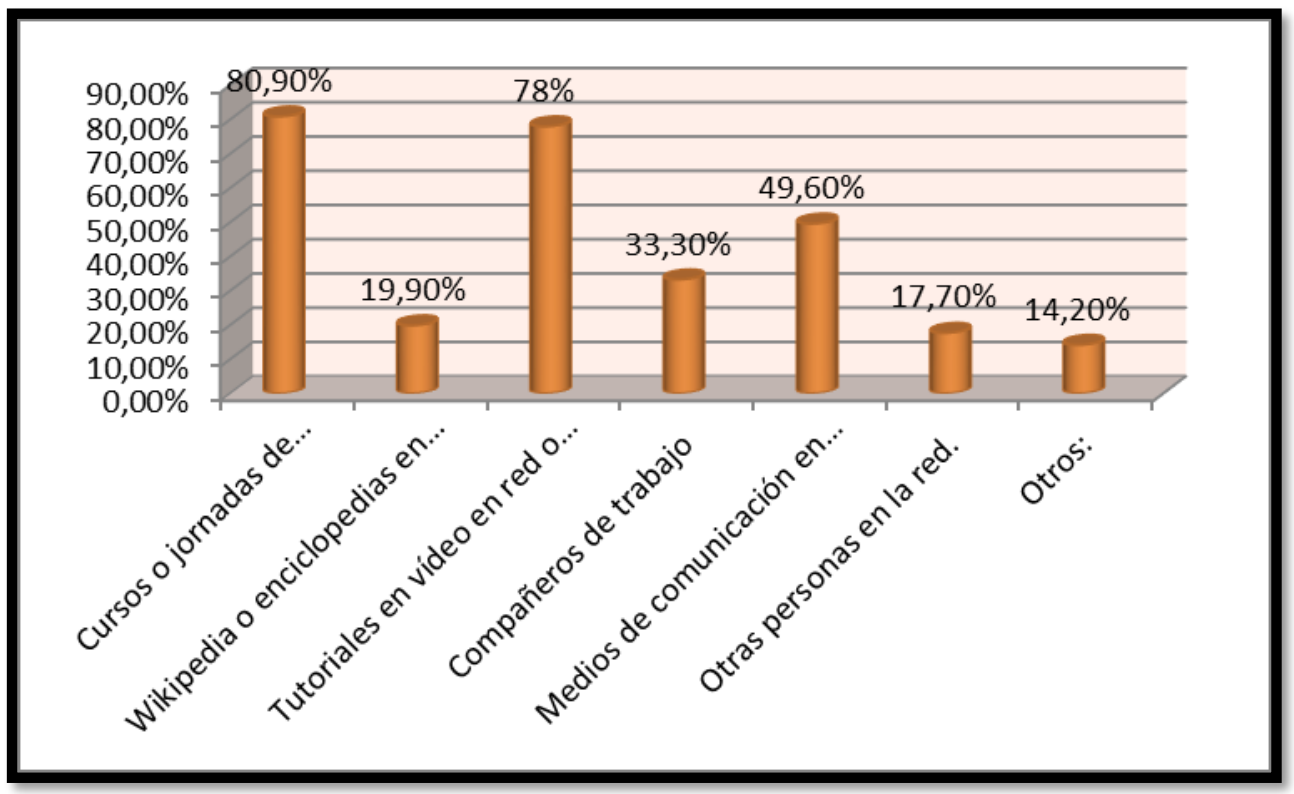

Figura 4. Limitaciones de las TIC

\section{CONCLUSIONES}

En la nueva sociedad del conocimiento, las TIC juegan un papel importante convirtiéndose en el eje central para el acceso a la información, esto trae consigo nuevos retos y cambios, no solo en la manera de pensar sino en la de actuar y ese cambio exige nuevas habilidades y desarrollo de nuevas competencias básicas de tipo tecnológico y que sean incluidas en la actividad docente. En Colombia la educación superior está expectante a grandes desafíos, entre ellos modificar el sistema educativo, incluyendo las TIC como un eje transversal del conocimiento y una competencia específica para el docente.

Las competencias TIC del docente universitario, son indispensables en los nuevos entornos de formación, por lo que es de suma importancia, realizar la valoración de dichas competencias que impliquen una adecuada formación y actualización, esto como una forma de adaptación a los cambios que surgen dentro del modelo educativo; la institución debe verificar que el docente se apropie de la valoración realizada y acepte los nuevos retos y desafíos que se presenten.

Luego de revisar y analizar la bibliografía y múltiples instrumentos de investigación, se evidenció que a la fecha, no había un investigación para conocer el estado de las competencias digitales de los docentes de la Unidades Tecnológicas de Santander, ni un instrumento diseñado y creado para tal fin, lo que justifica el desarrollo del presente estudio para conocer el estado de las competencias digitales en los profesores de la institución adaptando un instrumento ya utilizado y validado en otro estudio concreto, contextualizando este cuestionario al entorno Colombiano e institucional para determinar las necesidades de formación y generar una propuesta de mejora de las competencias consideradas básicas para un docente universitario de las UTS.

La información etnográfica arrojó que el $75 \%$ de los docentes se encuentran en rangos de edades que oscilan entre los 30 y 60 años, implicando que el total de estos docentes son inmigrantes o visitantes digitales, que a mayor edad, mayor es la tendencia de ir en contra del cambio de paradigma (Solano, Rocha, \& Marín, 2016) e incluir las competencias digitales como una competencia básica en la práctica docente.

La categoría o dimensión sobre uso y conocimiento de TIC permite establecer, la necesidad de capacitar a los docentes en el manejo de herramientas diferentes a las tradicionales ya que se evidenció un buen uso de las ofimáticas pero bajo uso y conocimiento de las herramientas de la web 2.0 e incluso de las 3.0 emergentes, que pueden fomentar el 
trabajo colaborativo y su vinculación en la actividad docente con apliaciones que pueden potenciar el uso de redes sociales en la docencia.

En la dimensión de integración de recursos en la práctica docente, se evidencia la carencia o falta en lo referente a estrategias metodológicas propias de la nueva era del conocimiento aunque existe un muy buen uso y conocimiento de las tradicionales, por lo que se hace necesario tenerlas en cuenta e incluirlas en el desarollo de las actividades académicas para incrementar la labor docente apoyadas con las nuevas herramientas de la información y la comunicación.

La innovación en TIC es muy baja, se evidencia poca particicpación en este tipo de proyectos que se apoyan en herramientas tecnológicas, así, como su preferencia en cuanto a su formación la prefieren en jornadas con cursos presenciales, aunque les interesa la formación de forma virtual como alternativa a la presencialidad.

Como una política o estrategia conducente a genererar una propuesta de mejora, es importante ofrecer acciones en las cuales se integren los conceptos y componentes básicos asociados a las TIC, así como ofrecer el conocimiento sobre herramientas telemáticas de información y comunicación, que potencien la formación del profesorado y la mejora de las competencias TIC. Las estrategias metodológicas deben fomentar el uso de TIC para el apoyo de la práctica docente incentivando el uso de herramientas en la cuales haya interacción con los alumnos; de la misma manera incentivar el uso de herramientas tecnológicas para la publicación de materiales educativos en la red de forma compartida y de libre acceso para los estudiantes y profesores, que redunden en una mejor práctica educativa de parte de los docentes para que sea replicada en el aula. Lo anterior hace conducente gestionar un programa de capacitación que incluya un seminario taller que supla las necesidades formativas detectadas teniendo en cuenta que la institución dentro de su propuesta formativa no cuenta con este tipo de oferta (Anexo A).

Como una limitación en la culminación del estudio, es la implementación de la propuesta de mejora, ya que depende de la Oficina de Desarrollo Académico (ODA), analizarla y dar el visto bueno para su implementación. La veracidad de los datos entregados por los profesores, aunque el cuestionario es anónimo se puede presentar sesgo dada la falta de veracidad en la información entregada por los docente, porque se perciben como "ignorantes" en el campo tecnológico y digital.

La implementación de la propuesta de mejora en la institución y el estudio realizado se presentan como base para una posible línea de trabajo doctoral, donde se pueda realizar una investigación sobre la influencia de la propuesta de mejora en los docentes, luego de su implementación y desarrollo, para poder verificar el nivel de apropiación de estas competencias.

\section{REFERENCIAS BIBLIOGRÁFICAS}

Agreda, M., Hinojo, M. A., \& Sola, J. M. (2016). Diseño y validación de un instrumento para evaluar la competencia digital de los docentes en la educación superior Española. Píxel-Bit. $\begin{array}{llll}\text { Revista } \quad \text { Medios } & \text { Educación(49), }\end{array}$ doi:http://dx.doi.org/10.12795/pixelbit.2016.i49.03

Bellei , C., Poblete, X., Sepúlveda, P., Orellana, V., \& Abarca , G. (2013). Situación educativa de América Latina y el Caribe: Hacia la educación de calidad para todos al 2015. Santiago de Chile, Chile: Imbunche Ltda. UNESCO-CEPAL, Oficina Regional de Educación para América Latina y el Caribe. Santiago: Ediciones del Imbunche . Obtenido de http://www.unesco.org/new/fileadmin/MULTIMEDIA/FIELD/Santiago/images/SITIEDespanol.pdf 
Blasco, J., Mengual, S., \& Roig, R. (2007). Competencias tecnológicas en el espacio europeo de educaciónsuperior. Propuesta de formación del maestro especialista en educación física. Profesorado: Revista de curriculum y formación del profesorado. Recuperado el 08 de 08 de 2018, de http://www.ugr.es/ recfpro/rev112ART10.pdf

Carrera, X., \& Coiduras, J. (2012). Identificación de la competencia dgital del profesor universitario: un estudio exploratorio en el ámbito de las Ciencias Sociales. Revista de Docencia Universitaria, 10(2), 273-298. Recuperado el 08 de 08 de 2018, de http://repositori.udl.cat/bitstream/handle/10459.1/47980/018608.pdf?sequence=1\&isAllowed $=\mathrm{y}$

Cerverá, M. (2013). Nuevos escenarios para los aprendices digitales en la universidad. Aloma: Revista de psicología, ciencias de la educación y el deporte, 1(31). Recuperado el 08 de 08 de 2018, de http://www.raco.cat/index.php/Aloma/article/viewFile/266714/354336

Cerverá, M., Martínez , J., \& Mon, F. (2015). Competencia digital y competencia digital docente: una panorámica sobre el estado de la cuestión. Revista Interuniversitaria de Investigación en Tecnología Educativa. Recuperado el 08 de 08 de 2018, de http://revistas.um.es/riite/article/download/257631/195811

Durán, M. (2014). Diseño y Validación de un instrumento de evaluación para la certificación de la competencia TIC del profesorado universitario. In Congreso Internacional Edutec 2014: El hoy y el mañana junto a las TIC. Recuperado el 08 de 08 de 2018, de https://digitum.um.es/xmlui/bitstream/10201/41373/1/TFM\%20Marta\%20Duran\%20Cuartero. pdf

Gisbert, M., \& Esteve, F. (2011). Digital Leaners: la competencia digital de los estudiantes universitarios. La Cuestión Universitaria(7), 48-59. Recuperado el 08 de 08 de 2018, de ISSN 1988-236x

Guil Bozal, M. (2005). Escala mixta likert-thurstone. Anduli - Revista Andaluza de Ciencias Sociales(5), 81-95. doi:http://dx.doi.org/10.12795/anduli

Hernández, C., Gamboa, A., \& Ayala, E. (2014). COMPETENCIAS TIC PARA LOS DOCENTES DE EDUCACION SUPERIOR. Congreso Iberoamericano de Ciencia, Tecnología, Innovación y Educación, 12, pág. 20. Buenos Aires. Recuperado el 08 de 08 de 2018, de https://www.oei.es/historico/congreso2014/memoriactei/837.pdf

Lengerke, O. (2017). Informe de gestión a 30 de Septiembre de 2017. Informe de gestión, Unidades Tecnológicas de Santander, Bucaramanga-Colombia. Obtenido de http://www.uts.edu.co/portal/files/Informe\%20de\%20gestionSeptiembre\%20de\%202017.pdf

Martínez, V. (2013). Paradigmas de investigación: manual multimedia para el desarrollo de trabajos de investigación. Una visión desde la epistemología dialectico crítica. Recuperado el 08 de 08 de 2018, de http://www.pics.uson.mx/wpcontent/uploads/2013/10/7_Paradigmas_de_investigacion_2013.pdf

Mortis, S., Váldes, A., Angulo, J., García, R., \& Cuevas , O. (2013). Competencias digitales en docentes de educación secundaria. Municipio de un estado del noroeste de México. Pesrpectiva Educacional, 52(2), 135-153. doi:10.4151/07189729-Vol.52-Iss.2-Art.174

Parlamento Europeo. (2006). Recomendación del parlamento europeo y del consejo del 18 de diciembre de 2006 sobre las competencias clave y el aprendizaje permanente. Parlamento Europeo. Diario Oficial de la Unión Europea. Obtenido de http://infofpe.cea.es/fpe/norm/Rec\%2018_2006.pdf

Pozuelo, J. (2014). ¿ Y si enseñamos de otra manera?. Competencias digitales para el cambio metodológico. Revista digital de investigación en docencia, 2(1), 21. Recuperado el 08 de 08 
de

2018 , de https://ebuah.uah.es/dspace/bitstream/handle/10017/20848/ense\%C3\%B1amos_Pozuelo_C ARACCIOLOS_2014_N2.pdf?sequence=1\&isAllowed $=y$

Prendes Espinoza, M., \& Gutiérrez Porlan, I. (2013). Competencias tecnológicas del profesorado en las universidades españolas. Revista de Educación, 196-222. doi:10.4438/1988-592X-RE-2011-361-140

Prendes, M. P. (2010). Competencias TIC para la docencia en la Universidad Pública Española: Indicadores y propuestas para la definición de buenas prácticas. Informe del Proyecto EA2009-0133 de la Secretaria de Estado de Universidades e Investigación, Universidad de murcia, Programa de Estudio y Análisis., Murcia. Recuperado el 08 de 08 de 2018, de //www. um. es/competenciastic/informe \_final\_competencias2010.pdf

Puentes, A., Roig, R., Sanhueza, S., \& Friz, M. (2013). Concepciones sobre las tecnologías de la información y la comunicación(TIC) y sus implicaciones educativas: Un estudio exploratorio con profesorado de la provincia de Ñuble, Chile. Revista CTS, 8(22), 75-88. Recuperado el 08 de 08 de 2018, de https://rua.ua.es/dspace/bitstream/10045/35183/1/2013_Puentes_etal_CTS.pdf

Quero Virla, M. (2010). Confiabilidad y Alpha de Cronbach. TELOS: Revista de estudios interdisciplinarios en ciencias sociales, 12(2), 248-252. Recuperado el 08 de 08 de 2018, de http://www.redalyc.org/pdf/993/99315569010.pdf

Rangel, A. (2015). COMPETENCIAS DOCENTES DIGITALES: PROPUESTA DE UN PERFIL. Píxel-Bit. Revista de Medios y Educación(46), 235-248. doi:doi: http://dx.doi.org/10.12795/pixelbit.2015.i46.15

Rangel, A., \& Peñalosa, E. (Julio de 2013). ALFABETIZACIÓN DIGITAL EN DOCENTES DE EDUCACIÓN SUPERIOR: CONSTRUCCIÓN Y PRUEBA EMPÍRICA DE UN INSTRUMENTO DE EVAlUACIÓN. Píxel-Bit. Revista de Medios y Educación(43), 9-23. doi:http://dx.doi.org/10.12795/pixelbit.2013.i43.01

Rocha V, A. R. (2018). Competencias digitales en los estudiantes. Tesis magistral, Universidad de Islas Baleares, Máster oficial en Tecnología Educativa E:Learning y gestión del conocimiento, Palma. Recuperado el 08 de 08 de 2018

Sanabria, A., \& Hernández, C. (2011). Percepción de los estudiantes y profesores sobre el uso de las TIC en los procesos de cambio e innovación en la enseñanza superior. Aloma: Revista de Psicologia, Ciències de l'Educació $i$ de l'Esport(29), 273-290. Obtenido de http://www.revistaaloma.net/index.php/aloma/article/view/106

Solano, E., Rocha, A. R., \& Marín, V. (2016). ). Instrumentos de Investigación en Tecnología Educativa: Una aproximación a los instrumentos validados de competencia digital. CIINATIC Congreso Internacional de innovación y Apropiación de las Tecnologías de la Información y la Comunicación. Bucaramanga. Recuperado el 08 de 08 de 2018, de http://media.wix.com/ugd/6e2c11_383068db09a94b24a044201c95421c50.pdf

Torres, T. (2014). Competencia dígital del profesorado de educación secundaria: un instrumento de evaluación. Tesis de Máster. , Universidad de Murcia, MÁSTER INTERUNIVERSITARIO TECNOLOGÍA EDUCATIVA: E-LEARNING Y GESTIÓN, Murcia. Recuperado el 08 de 08 de https://digitum.um.es/jspui/bitstream/10201/40351/1/\%2522TFM_TORRES\%20ALCANTARA .pdf

Valdivieso, T., \& González, M. (2016). Competencia digital docente: ¿Dónde estamos?. Perfil del docente de educación primaria y secundaria. El caso de Ecuador. Píxel-Bit. Revista de Medios y Educación(49), 57-73. doi:http://dx.doi.org/10.12795/pixelbit.2016.i49.04 
Zabalza, M., \& Beraza, M. (2003). Competencias docentes del profesorado universitario: calidad y desarrollo profesional. (Vol. 4). Narcea Ediciones. Recuperado el 08 de 08 de 2018,

https://books.google.es/books?id=ho6AanfMHy8C\&dq=Competencias+docentes+del+profes orado+universitario:+calidad+y+desarrollo+profesional\&lr=\&hl=es\&source=gbs_navlinks_s

\section{ANEXO A}

\section{PROPUESTA DE MEJORA DE LAS COMPETENCIAS DIGITALES}

El objetivo del seminario taller es capacitar a los docentes de las Unidades Tecnológicas de Santander en el conocimiento y manejo de aplicaciones de tipo colaborativo para adquirir la competencias básicas en TIC, que apoyen el proceso académico, investigativo y de gestión administrativa en la institución.

El contenido del seminario taller se estructura de la siguiente forma:

1. Herramientas para la comunicación

$>$ Correo electrónico

$>$ Foros

$>$ Redes Sociales

2. Herramientas para compartir, seleccionar y organizar fuentes de información en Internet

$>$ Marcadores sociales y organizadores de enlaces

$>$ Agragadores de fuente RSS

$>$ Sistemas de gestión de alertas

> Herramientas para la curación de contenidos

$>$ Herramientas para la detección de plagio

3. Trabajo con archivos en la nube (Cloud Computing)

$>$ Aplicaciones ofimáticas en línea

> Gestión de archivos en la nube

4. Espacios virtuales de trabajo

$>$ Redes sociales para trabajo colaborativo

$>$ Gestión de proyectos y tareas compartidas

$>$ Multimedia Sharing

5. Herramientas para presentación de resultados

$>$ Plataformas de Blogs y microblogs

$>$ Wikis

$>$ Herramientas para presentaciones

La tabla 3 muestra de forma específica cada una de las actividades y herramientas a utilizar en el seminario taller 
Tabla 3 Actividades a realizar propuesta de mejora

\begin{tabular}{|c|c|c|c|c|}
\hline SESIÓN & CONTENIDO & ACTIVIDAD & $\begin{array}{l}\text { INTENSIDAD } \\
\text { HORARIA }\end{array}$ & \\
\hline $\begin{array}{l}\text { HERRAMIENTAS } \\
\text { PARA LA } \\
\text { COMUNICACIÓN }\end{array}$ & $\begin{array}{l}\text { Correo electrónico } \\
\text { Foros } \\
\text { Redes Sociales }\end{array}$ & $\begin{array}{l}\text { Conceptos Básicos } \\
\text { Introducción a la Web }\end{array}$ & 1 & \multirow{3}{*}{ SEMANA 1} \\
\hline \multirow{4}{*}{ 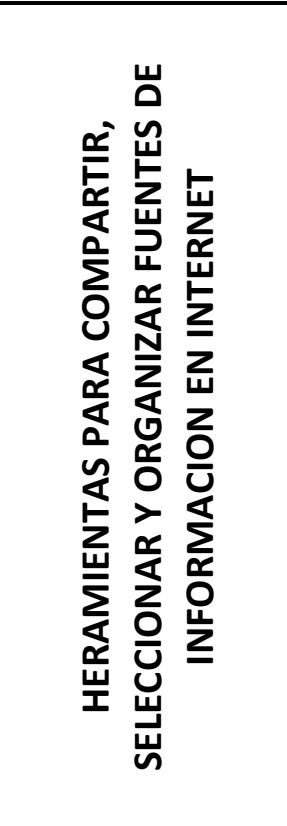 } & $\begin{array}{l}\text { Marcadores Sociales y } \\
\text { Organizadores de } \\
\text { Enlaces }\end{array}$ & $\begin{array}{l}\text { Delicious } \\
\text { Diigo } \\
\text { Evernote }\end{array}$ & 2 & \\
\hline & $\begin{array}{l}\text { Agregadores de } \\
\text { Fuentes RSS }\end{array}$ & $\begin{array}{l}\text { NetVibes } \\
\text { Google Reader } \\
\text { Feedly } \\
\text { Feed Reader } \\
\text { RssOwl } \\
\end{array}$ & 3 & \\
\hline & $\begin{array}{l}\text { Sistemas de gestión } \\
\text { de alertas }\end{array}$ & $\begin{array}{l}\text { Google alerts otras } \\
\text { extensiones Google } \\
\text { chrome y FireFox }\end{array}$ & 1 & \multirow{3}{*}{ SEMANA 2} \\
\hline & $\begin{array}{l}\text { Herramientas para la } \\
\text { curación de } \\
\text { contenidos }\end{array}$ & $\begin{array}{l}\text { Scoop.it } \\
\text { Buffer } \\
\text { Flipboard } \\
\text { Pocket }\end{array}$ & 4 & \\
\hline \multirow{3}{*}{ 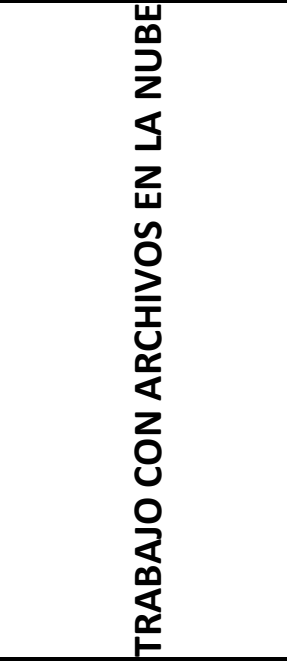 } & $\begin{array}{l}\text { Conceptos básicos } \\
\text { sobre cloud } \\
\text { computing } \\
\end{array}$ & & 1 & \\
\hline & $\begin{array}{l}\text { Aplicaciones } \\
\text { ofimaticas en línea }\end{array}$ & $\begin{array}{l}\text { Zoho } \\
\text { Microsoft Office Web } \\
\text { Apps } \\
\text { ThinkFree Office } \\
\text { Google Docs } \\
\end{array}$ & 3 & \multirow[b]{2}{*}{ SEMANA 3} \\
\hline & $\begin{array}{l}\text { Gestión de archivos } \\
\text { en la nube }\end{array}$ & $\begin{array}{l}\text { DropBox } \\
\text { Google Drive } \\
\text { One Drive } \\
\text { Ubuntu One } \\
\text { ICloud }\end{array}$ & 3 & \\
\hline \multirow{3}{*}{ 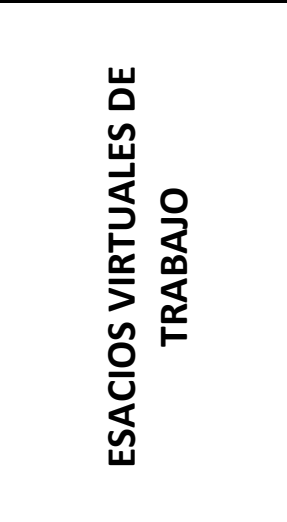 } & $\begin{array}{l}\text { Redes sociales para el } \\
\text { trabajo en equipo }\end{array}$ & $\begin{array}{l}\text { Facebook } \\
\text { Twitter } \\
\text { Google }+ \\
\text { Research Gate } \\
\text { Linkedin } \\
\end{array}$ & 3 & \multirow[t]{2}{*}{ SEMANA 4} \\
\hline & $\begin{array}{l}\text { Gestión de proyectos } \\
\text { y tareas compartidas }\end{array}$ & $\begin{array}{l}\text { Google Calendar } \\
\text { Zoho } \\
\text { TeamBox } \\
\end{array}$ & 3 & \\
\hline & Multimedia Sharing & $\begin{array}{l}\text { Usos legales de la } \\
\text { producción digital }\end{array}$ & 3 & SEMANA 5 \\
\hline
\end{tabular}




\begin{tabular}{|c|c|c|c|c|}
\hline & & $\begin{array}{l}\text { Imagen: gestión de } \\
\text { imágenes y servicios } \\
\text { online } \\
\text { Vídeos: Youtube, Vimeo } \\
\text { Audio: Ivoox } \\
\text { Herramientas para } \\
\text { detectar plagio: } \\
\text { ThePlagiarismChecker, } \\
\text { Plagiarisma.net } \\
\end{array}$ & & \\
\hline \multirow{3}{*}{ 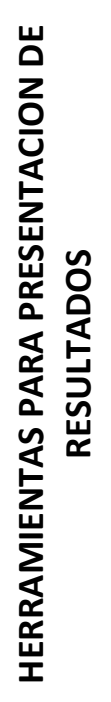 } & $\begin{array}{l}\text { Plataformas de blogs y } \\
\text { microblogs }\end{array}$ & \begin{tabular}{|l} 
Blogger \\
Wordpress \\
Tumblr \\
Hypotheses.org \\
\end{tabular} & 2 & \\
\hline & Wikis & $\begin{array}{l}\text { Wikihow } \\
\text { Pbworks } \\
\text { Hackpad }\end{array}$ & 1 & \\
\hline & $\begin{array}{l}\text { Herramientas para } \\
\text { presentaciones }\end{array}$ & $\begin{array}{l}\text { Crear presentaciones: } \\
\text { Prezi, Present.me, Emaze, } \\
\text { Keynote, Zoho } \\
\text { presentation. } \\
\text { Compartir } \\
\text { presentaciones: Issuu, } \\
\text { Slideshare, Slidetalk, } \\
\text { Scribb } \\
\end{array}$ & 6 & SEMANA 6 \\
\hline
\end{tabular}

La duración establecida, para llevar a cabo la capacitación consiste en 54 horas de trabajo de las cuales 36 son de trabajo presencial en aula y 18 de trabajo virtual, dividida en 6 sesiones semanales de 3 horas, dos días a la semana.

La metodología es presencial en el aula, apoyo de palataforma MOODLE, para realizar la entrega de las evidencias y productos que incluye ejercicios individuales, lecturas de apoyo participación en foros. La sesión de trabajo presencial será explicativa sobre el manejo básico de las herramientas y aclaración sobre conceptos fundamentales, así como ejercicios de aplicación.

\section{INFORMACIÓN SOBRE LOS AUTORES}

\section{Ernesto Solano Hernández}

\section{Unidades Tecnológicas de Santander (UTS)}

Ingeniero de Sistemas egresado de la Universidad Industrial de Santander, estudió un diplomado en redes de la Universidad Autónoma de Bucaramanga y el instituto Tecnológico de estudios superiores de Monterrey; Máster en Tecnología Educativa y generación de conocimiento de la Universidad de Islas Baleares en España. Actualmente cursa estudios de doctorado en Universidad de Islas Baleares. Es miembro del grupo CoVIF comunidad virtual de investigadores en formación de la universidad de Islas Baleares (España). Miembro del grupo GRIIS, Grupo de Investigación en Ingeniería del Software de las Unidades Tecnológicas de Santander.

Interés principal de investigación es la de formador de formadores en tecnologías de la información y la comunicación TIC, competencia digital, recursos educativos digitales; otros intereses investigativos como: lógica, algoritmos, e-learning, plataformas virtuales, uso de software libre, diseño de recursos educativos digitales 
Conocimientos en E-learning, Programación estructurada, paradigma de programación orientada a objetos, lógica de programación, sistemas operativos, bases de datos

\author{
Victoria I. Marín Juarros \\ Carl von Ossietzky Universität Oldenburg (Alemania)
}

Licenciada en Pedagogía, especialización de Formación en la Organización, Máster en Tecnología Educativa: e-learning y gestión del conocimiento y Doctora en Tecnología Educativa: Aprendizaje y Gestión del Conocimiento. Miembro investigador del Grupo de Tecnología Educativa de la UIB (GTE). Actualmente investigadora postdoctoral en la Carl von Ossietzky Universität Oldenburg de Alemania.

Sus intereses investigadores son entre otros: los entornos personales de aprendizaje (PLE), la formación inicial del profesorado, el e-learning, medios sociales en la formación y desarrollo profesional, software libre en educación, redes sociales académicas, el aprendizaje móvil, eportfolios en educación superior, aprendizaje autorregulado con TIC, aprendizaje basado en la investigación con herramientas digitales.Web: http://vmarinj.wordpress.com

\title{
Alba Rossi Rocha Vásquez \\ Unidades Tecnológicas de Santander (UTS)
}

Alba Rossi Rocha Vásquez es Ingeniera de Sistemas egresada de la Universidad Industrial de Santander, diplomada en redes de la Universidad Autónoma de Bucaramanga y el Instituto Tecnológico de Estudios Superiores de Monterrey; Máster en Tecnología Educativa: E-learning y gestión de conocimiento de la Universidad de Islas Baleares en España. Es profesora tiempo completo adscrita a la facultad de ciencias naturales e ingeniería de las Unidades Tecnológicas de Santander. Coordinadora del repositorio institucional de las Unidades Tecnológicas de Santander (RIUTS). Su línea de investigación es la de. Es miembro del grupo CoVIF comunidad virtual de investigadores en formación de la universidad de Islas Baleares (España). Miembro de la dirección de investigaciones y extensión UTS. Miembro del grupo GRIIS, Grupo de Investigación en Ingeniería del Software de las Unidades Tecnológicas de Santander.

Su interés principal de investigación es la de Efectos de los avances tecnológicos en el proceso de enseñanza-aprendizaje, competencia digital, recursos educativos digitales; otros intereses investigativos como: lógica, algoritmos, e-learning, plataformas virtuales, uso de software libre, diseño de recursos educativos digitales

Conocimientos en E-learning, Programación estructurada, paradigma de programación orientada a objetos, lógica de programación, sistemas operativos, bases de datos, gestión documental.

Los textos publicados en esta revista están sujetos a una licencia de Reconocimiento 4.0 España de Creative Commons. Puede copiarlos, distribuirlos, comunicarlos públicamente y hacer obras derivadas siempre que reconozca los créditos de las obras (autoría, nombre de la revista, institución editora) de la manera especificada por los autores o por la revista. La licencia completa se puede consultar en:Licencia Creative Commons Atribución-NoComercial-Compartir por igual 4.0 Internacional. 